\title{
Photometric properties of dwarf galaxies in the Coma cluster: radial dependence
}

\author{
Yutaka Komiyama ${ }^{1}$, Sadanori Okamura ${ }^{2}$, Masafumi Yagi $^{3}$, \\ Nobunari Kashikawa ${ }^{3}$, Bianca M. Poggianti ${ }^{4}$, Terry J. Bridges ${ }^{5}$, \\ Bahram Mobasher ${ }^{6}$ and Dave Carter ${ }^{7}$ \\ ${ }^{1}$ Subaru Telescope, Hilo, HI, USA email: komiyama@subaru.naoj.org \\ ${ }^{2}$ Department of Astronomy, University of Tokyo, Bunkyo-ku, Tokyo, Japan \\ ${ }^{3}$ National Astronomical Observatory of Japan, Mitaka, Tokyo, Japan \\ ${ }^{4}$ Osservatorio Astronomico di Padova-INAF, Padova, Italy \\ ${ }^{5}$ Department of Physics, Queen's University, Kingston, Canada \\ ${ }^{6}$ Space Telescope Science Institute, Baltimore, MD, USA \\ ${ }^{7}$ Liverpool John Moores University, Birkenhead, Wirral, UK
}

\begin{abstract}
Photometric properties (effective surface brightness, effective radius, radial profile index, axis ratio, color, color gradient) of 328 galaxies in the Coma cluster fainter than $R=15$ mag are examined as a function of the distance from the cluster center. No significant gradient is found for the effective surface brightness, effective radius and radial profile index. The distribution of axis ratios shows a concentration of round galaxies at the cluster center in the magnitude range $16.5<R<18$; most of these are found to be old and to have intermediate metal abundance, suggesting that they are nucleated dwarf ellipticals. On the other hand, we find a significant gradient in color, in the sense that galaxy colours become bluer with increasing distance from the cluster center. We conclude that this color gradient represents a metallicity gradient.
\end{abstract}

\section{The data}

We carried out a wide-field imaging and spectroscopic survey in the Coma cluster using the Mosaic CCD Camera II and WYFFOS multi-fibre spectrograph on the William Herschel Telescope, obtaining homogeneous images for a $2.22 \mathrm{deg}^{2}$ area to $R \simeq 23 \mathrm{mag}$, and medium resolution spectra for about 500 galaxies to $R=20$ (Komiyama et al. 2002; Mobasher et al. 2001; Poggianti et al. 2001). In this study, we analyze the photometric properties of 328 spectroscopically confirmed cluster galaxies fainter than $R=15$ mag (121, 124 and 83 in magnitude ranges of $15<R<16.5,16.5<R<18$ and $18<R$, respectively).

\section{Radial dependence}

Photometric parameters are examined as a function of distance from the cluster center. We fit the distribution of a given parameter $P$ with $P=C \log d+D$, where $d$ is the distance from the cluster center, to see if there is a radial gradient. We assume that the radial gradient is significant when $C$ is larger than $2 \sigma_{C}$, where $\sigma_{C}$ is the fitting error of C. 


\subsection{Luminosity structure parameters: $\mu_{e}, r_{e}, N$, axis ratio}

We first examine the luminosity structure parameters and find no significant radial gradient for the effective surface brightness $\left(\mu_{e}\right)$, effective radius $\left(r_{e}\right)$ and radial profile index $(N)$ fitted to the Sersic law $I(r)=I_{0} \exp \left[-\left(r / r_{0}\right)^{N}\right]$, in any magnitude range.

But we find a significant gradient of axis ratio for galaxies in the magnitude range $16.5<R<18$ (3.6 $\sigma$ significance), in the sense that round galaxies preferentially reside in the cluster center. The spectral analysis shows that most of these are old $(>9 \mathrm{Gyr})$ and have a typical metal abundance $(-1<Z<-0.15)$ for galaxies in this magnitude range. Ferguson \& Sandage (1989) showed that nucleated dwarf ellipticals in the Virgo and Fornax clusters tend to have round shapes and reside preferentially in the central region of the cluster. Therefore, round galaxies found in the center of Coma could be nucleated dwarf ellipticals. Their absolute magnitudes (roughly $-18<M_{R}<-16.5$ ) also support this idea. However, we cannot verify this because the nuclei are buried in the seeing disk in our images.

\subsection{Stellar population parameter: $B_{K}-R_{K}$}

We find that the $B_{K}-R_{K}$ color shows a significant gradient in all magnitude ranges (2.5, 2.8 and $4.5 \sigma$ significance for $15<R<16.5,16.5<R<18$ and $18<R$, respectively), in the sense that galaxies which are located at the outskirts of the cluster have bluer colors as seen in the left panels of Fig. 1. It is also noted that the slope of the gradient becomes steeper as galaxies become fainter.

Carter et al. (2002) reported that there is clear evidence of a metallicity gradient for the galaxies in the Coma cluster, based on the same data used in this study. However, their analysis included all galaxies, regardless of magnitude. We hence examine the metallicity distribution of galaxies in each magnitude range, in the same manner as we did for color. As seen in the right panels of Fig. 1, we find gradients in the metallicity distribution, although the significance levels are not as high as those for color $(2.4,0.9$ and $2.1 \sigma$ for $15<R<16.5,16.5<R<18$ and $18<R$, respectively). Since no significant gradient in the age distribution is found in all magnitude ranges, we conclude that the gradient in color represents a metallicity gradient, not an age gradient. We also note that the slope of the metallicity gradient is steeper for fainter galaxies, which is also the case for color.

We suggest that the metallicity gradient can be explained by pressure confinement by the hot intracluster medium (Babul \& Rees 1992). According to this scenario, a galaxy located in the inner regions of the cluster, where the pressure of the intracluster medium is high, would have a longer star-forming period and a higher metallicity compared to a galaxy in the outskirts of the cluster. In addition, since the time scale of gas confinement is partly determined by the binding energy (i.e. mass), massive galaxies would be more metal-rich than less massive galaxies for the same external pressure. This may account for the trend that the slope of the metallicity gradient becomes steeper for fainter galaxies.

\subsection{Color gradients within cluster galaxies: $d\left(B_{K}-R_{K}\right) / d r$}

The color gradients within galaxies show a significant variation across the cluster, for the faintest galaxies $(18<R)$. For this faint subsample, galaxies near the cluster center tend to have positive color gradients $(\Delta(\mathrm{B}-\mathrm{R}) / \Delta \log (\mathrm{r})>0$; i.e. colors become redder with increasing galactocentric distance), while galaxies near the cluster outskirts tend to have negative color gradients $(\Delta(\mathrm{B}-\mathrm{R}) / \Delta \log (\mathrm{r})<0$; i.e. colors become bluer with increasing galactocentric distance). This correlation is significant at a level of $3.3 \sigma$; no correlation is seen for brighter galaxies. The observed trend could reflect environmental effects for the evolution of dwarf galaxies, although the scatter is large. Further theoretical and observational studies are required to explain the nature of this trend. 

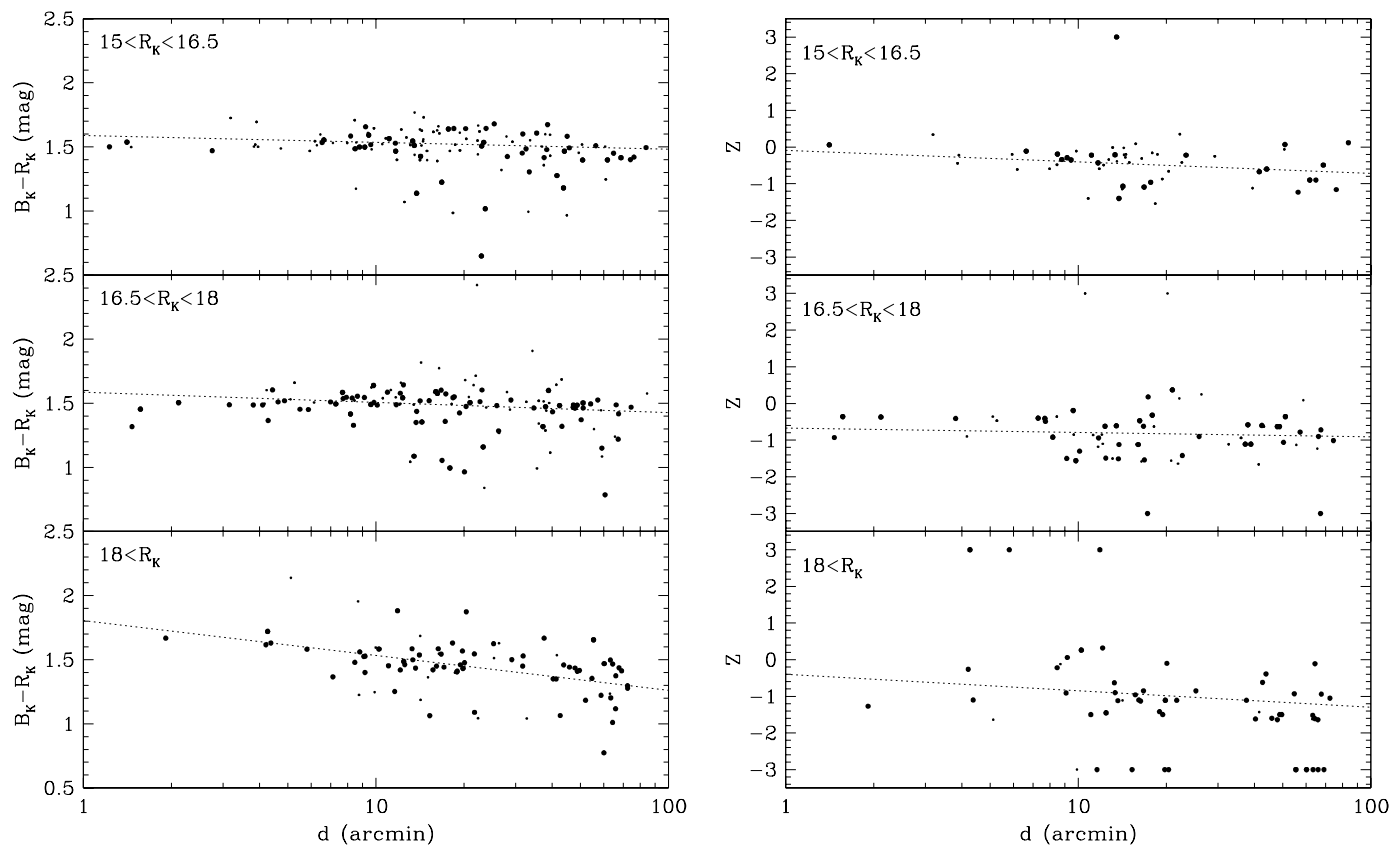

Figure 1. Left: $B_{K}-R_{K}$ color plotted as a function of the distance from the center of the Coma cluster. Isolated galaxies are plotted as large dots, while blended galaxies are plotted as small dots. The dotted lines show the best-fit lines for the data (overlying points are excluded from the fit). Right: Metallicity $Z$ plotted as a function of the distance from the cluster center. For galaxies lying outside the age-metal model grid, an arbitrary metallicity ( +3 or -3 dex) is assigned, but these galaxies are excluded from the fitting procedure.

\section{References}

Babul, A. \& Rees, M. J. 1992 MNRAS 255, 346

Carter D., Mobasher B., Bridges, T. J. et al. 2002 ApJ 567, 772

Ferguson, H. C. \& Sandage, A. 1989 ApJ 346, L53

Komiyama Y., Sekiguchi, M., Kashikawa, N. et al. 2002 ApJS 138, 265

Mobasher B., Bridges, T. J., Carter, D. et al. 2001 ApJS 137, 279

Poggianti B. M., Bridges, T. J., Mobasher, B. et al. 2001 ApJ 562, 689 